\title{
The prokaryotic expression, polyclonal antibody preparation, and subcellular localization of the transmembrane protein NS2A of the duck Tembusu virus
}

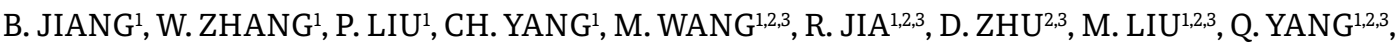 \\ Y. WU $U^{1,2,3}$, X. ZHAO ${ }^{1,2,3}$, A. CHENG ${ }^{1,2,3}$, S. CHEN ${ }^{1,2,3^{*}}$
}

\begin{abstract}
${ }^{1}$ Institute of Preventive Veterinary Medicine, Sichuan Agricultural University, Chengdu, No. 211 Huimin Road, Wenjiang District, Chengdu city, Sichuan province 611130, P. R. China; ${ }^{2}$ Research Center of Avian Disease, College of Veterinary Medicine, Sichuan Agricultural University, Chengdu, Sichuan 611130, P. R. China; ${ }^{3}$ Key Laboratory of Animal Disease and Human Health of Sichuan Province, Sichuan Agricultural University, Chengdu, Sichuan 611130, P. R. China
\end{abstract}

Received June 30, 2019; revised December 9, 2019; accepted August 10, 2020

\begin{abstract}
Summary.-Duck Tembusu virus (DTMUV) is a single-stranded, positive-sense RNA arbovirus, belonging to the genus Flavivirus, the family Flaviviridae. As a transmembrane protein, non-structural protein 2A (NS2A) plays an important role in virion assembly, replication complex and antagonizing host immune response. Since NS2A protein contains many hydrophobic amino acids, it is hard to gain the full-length protein of NS2A for prokaryotic expression. Therefore, to make a deep study, prokaryotic expression and polyclonal antibody preparation of truncated DTMUV NS2A was performed. The truncated NS2A gene $\left(178-450 \mathrm{bp}\right.$ ) was obtained, and sub-cloned into the prokaryotic vector pGEX-4T-1 (pGEX-4T-1-NS2A $\left.\mathrm{A}_{178-450 \mathrm{bp}}\right)$. Subsequently, the recombinant GST-NS2 $\mathrm{A}_{60-150 a a}$ protein was successfully expressed in E. coli BL21 (DE3) with the induction by $0.3 \mathrm{mmol} / \mathrm{l}$ isopropyl $\beta$-D-thiogalactoside (IPTG) for $6 \mathrm{~h}$ at $37^{\circ} \mathrm{C}$. The GST-NS2A $60-150 \mathrm{aa}$ protein was extracted from the gel. The BALB/c mice were immunized with the purified recombinant NS2A protein to prepare polyclonal antibodies against the truncated NS2A protein. The titer of the polyclonal antibodies, determined by ELISA analysis, was 1:128,000. The specificity of the polyclonal antibodies (mPAb-DTMUV-NS2A $\mathrm{A}_{60-150 a}$ ) were verified by Western blot analysis. Furthermore, the indirect immunofluorescence (IFA) was performed to explore the subcellular localization of NS2A. NS2A protein was, in the transfected cells, located mainly around nucleus in the endoplasmatic reticulum. Taken together, our study provided a useful tool for the further exploration of the biological functions and molecular mechanism of DTMUV NS2A.
\end{abstract}

Keywords: duck Tembusu virus; non-structural protein 2A; prokaryotic expression; polyclonal antibodies; subcellular location

Duck Tembusu virus (DTMUV) is an emerging member of the genus Flavivirus within the family Flaviviridae (Bhatt et al.,2013; Zhang et al., 2017). The DTMUV outbreak, in 2010, in China caused egg-drop of almost 120 million

"Corresponding author. E-mail: shunchen@sicau.edu.cn; phone: +86-028-8629-1482.

Abbreviations: DENV = dengue virus; DTMUV = duck Tembusu virus, $\mathrm{ER}=$ endoplasmatic reticulum; NS2A = non-structural protein $2 \mathrm{~A} ; \mathrm{TMD}=$ transmembrane domain ducks and infected 15 million meat ducks, resulting in serious economic loss of several billion dollars in the Chinese poultry industry (Su et al., 2011). In addition to duck, DTMUV can infect also many other birds, such as chickens, geese and house sparrows (Liu et al., 2012; Tang et al., 2013; Wang et al., 2016). In previous study, it was proved that DTMUV can infect many mammalian cells including, HEK293, BHK21, Vero cells (Shaozhou et al., 2015; Sun et al., 2017; Wang et al., 2016) and duck embryo fibroblasts (DEF). The DTMUV genome, consists of three structural proteins (C, prM and E) and seven non-structur- 
(a)

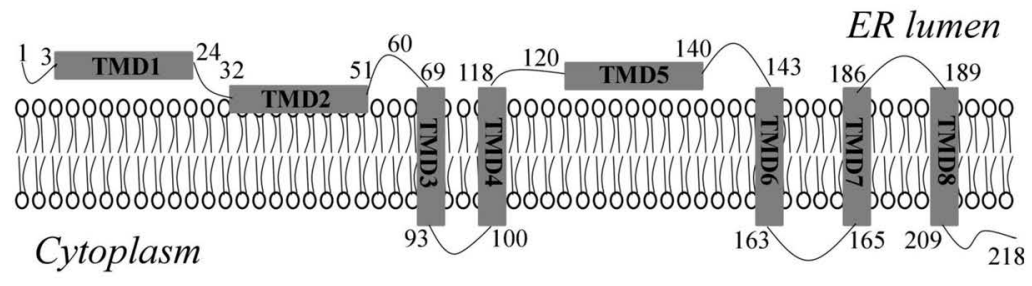

(b)

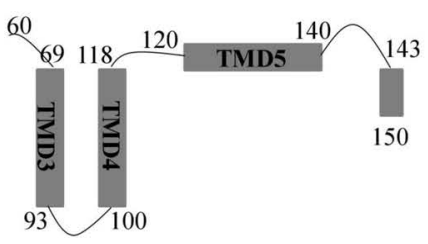

(c)

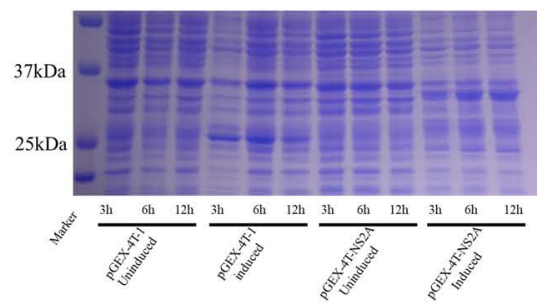

(d)

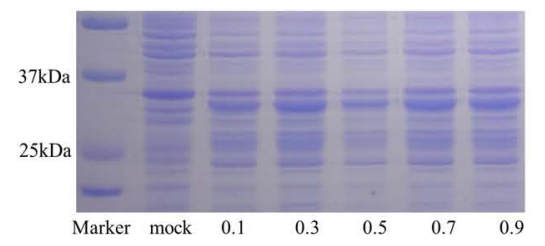

(e)

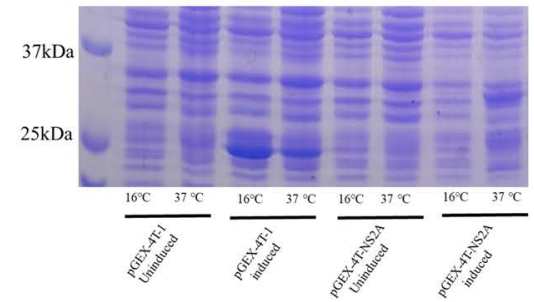

\section{Fig. 1}

Membrane topology of NS2A and optimization analysis of the expression conditions of the NS2A ${ }_{60-150 a a}$ protein

A topology model of DENV-NS2A on the ER membrane was obtained from previous study (a) and model of our truncated DTMUV NS2A (b). The truncated NS2A protein optimization of the induction time, $3 \mathrm{~h}, 6 \mathrm{~h}$ and $12 \mathrm{~h}$ (c), optimization of IPTG concentration (d) and optimization of the induction temperature (e).

al proteins (NS1, NS2A, NS2B, NS3, NS4A, NS4B and NS5). As a transmembrane protein, non-structural protein $2 \mathrm{~A}$ (NS2A) is an important component of replication complex and is associated with the endoplasmic reticulum (ER). As reported, NS2A has 8 transmembrane domains (TMDs) which help NS2A to anchor to ER membrane. Flavivirus NS2A is a hydrophobic protein with a size of approximately $22 \mathrm{kDa}$ (Chambers et al., 1989). It has been shown that NS2A takes part in the membrane rearrangements and helps to the rearrangement of four non-structural proteins: NS2A, NS2B, NS4A and NS4B, which take part in the construction of replication complex (Westaway et al., 1997). In previous study, NS2A has been found to possess many functions also in other flaviviruses. For example, NS2A takes part in the replication of the virus and has an essential role in virion assembly (Kummerer and Rice, 2002; Leung et al., 2008; Xie et al., 2013). In addition, NS2A antagonizes the host immune response (Liu et al., 2004; Liu et al., 2006; Munoz-Jordan et al., 2003). Furthermore, NS2A has been proved to take part in the production of infectious particles (Chambers et al., 1989; Tu et al., 2012). However, there are only few studies about DTMUV NS2A. Therefore, to further study the function of DTMUV NS2A, prokaryotic expression and polyclonal antibody preparation of NS2A were performed in this study.
Because, TMDs contain many hydrophobic amino acids and rare codes, we were not able to obtain the full-length protein of NS2A. Thus, in our research, the recombinant

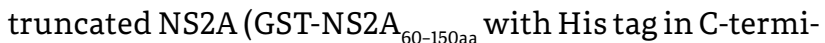
nal) was purified and used to immunize mouse for the preparation of polyclonal antibody. Finally, we obtained mouse-anti NS2A polyclonal antibodies (mPAb-DTMUVNS2A $_{60-150 a a}$ ), with high titer determined by enzyme-linked immunosorbent assay (ELISA). Additionally, the localization of NS2A was detected by indirect immunofluorescence (IFA) with mPAb-DTMUV-NS2A $\mathrm{A0-150aa}$.

In our study, a sensitive and specific polyclonal antibody (mPAb-DTMUV-NS2A ${ }_{60-150 a a}$ ) was used to detect the localization of NS2A in the DTMUV infected DEF, which provides a basis for further exploration of the biological functions of DTMUV NS2A.

According to the previous report, a topology model of DENV-NS2A on the ER membrane was obtained (Fig. 1a) (Sun et al., 2017). To obtain the antigen, we have used truncated NS2A (60-150 aa), as shown in Fig.1b. The sequence of this fragment was amplified by PCR and inserted into pGEX4T-1 vector. The recombinant plasmid was transformed into Escherichia coli BL21 (DE3) cells. Bacterial colonies were screened by PCR and the positive colonies were sequenced. 
(a)

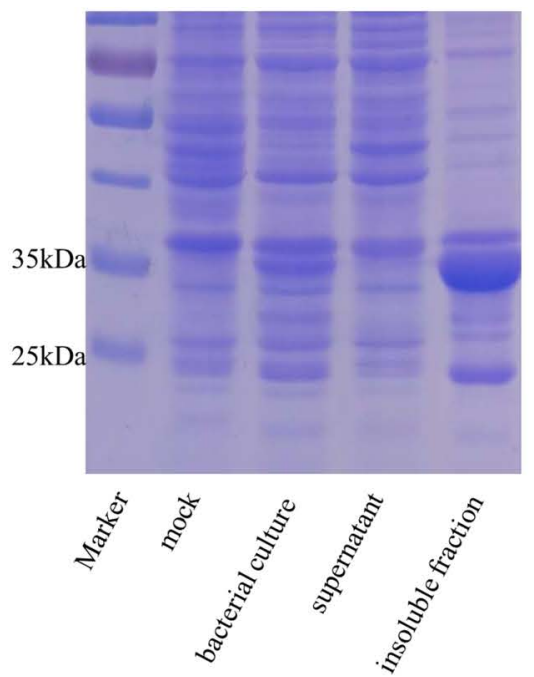

(b)

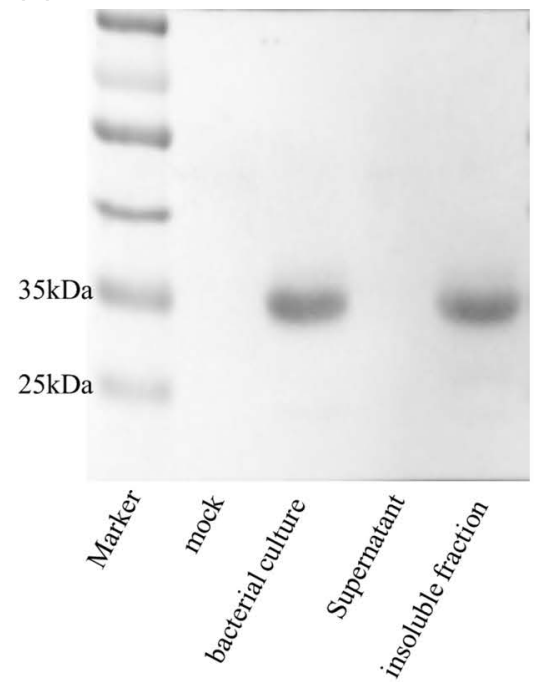

(c)

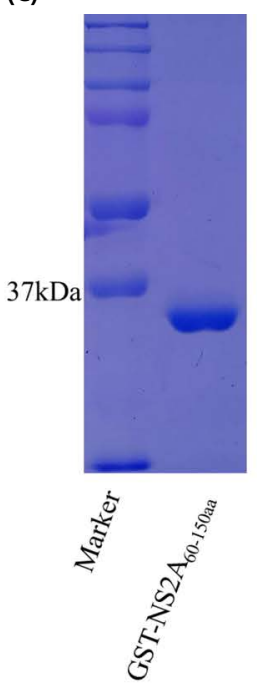

(d)

$37 \mathrm{kDa}$
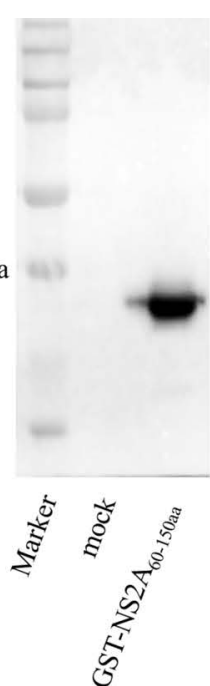

Fig. 2

SDS-PAGE and Western blot of the recombinant GST-NS2A

SDS-PAGE of bacterial culture and lysates stained with Coomasie brilliant blue (a). Detection of GST-NS2A 60-150aa by Western blot analysis with the mouse anti-His antibody (b). Mock: negative control without recombinant protein; bacterial culture: culture containing recombinant NS2A protein before lysis; supernatant: supernatant of the sonicated GST-NS2A lysate; insoluble fraction: insoluble fraction of the sonicated GST-NS2A. SDS-PAGE of the purified recombinant protein GST-NS2A stained with Coomasie brilliant blue (c). Detection of purified recombinant protein GST-NS2A by Western blot analysis with the mouse anti-His antibody (d).

(a)

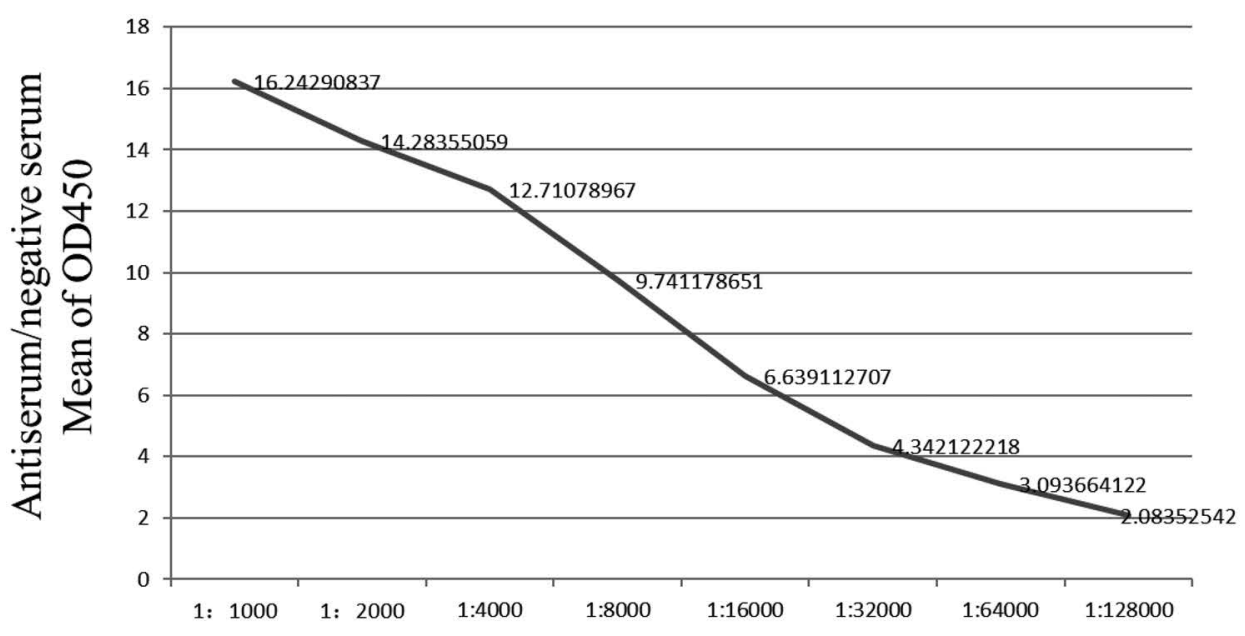

Dilution of serum (b)
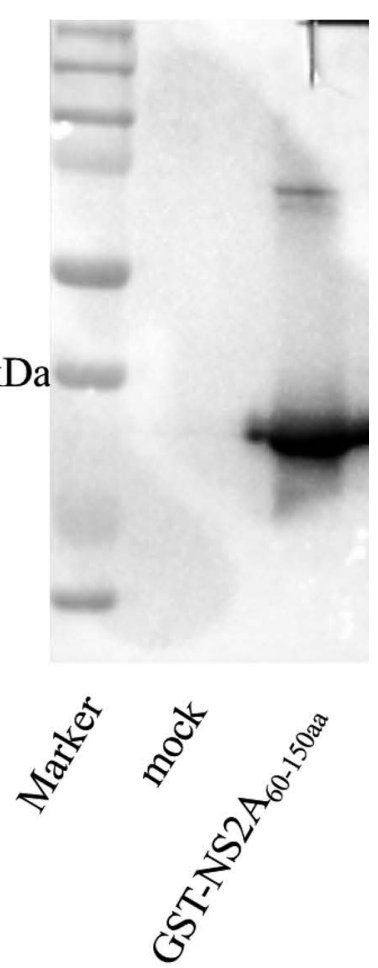

Fig. 3

Titer determination of polyclonal antibodies by ELISA and Western blot analysis of the purified recombinant NS2A protein The titer of anti-serum was determined by ELISA (a). Western blot analysis of the purified recombinant non-structural protein NS2A with polyclonal anti-serum, lane 1: negative control; lane 2: purified recombinant protein (b). 

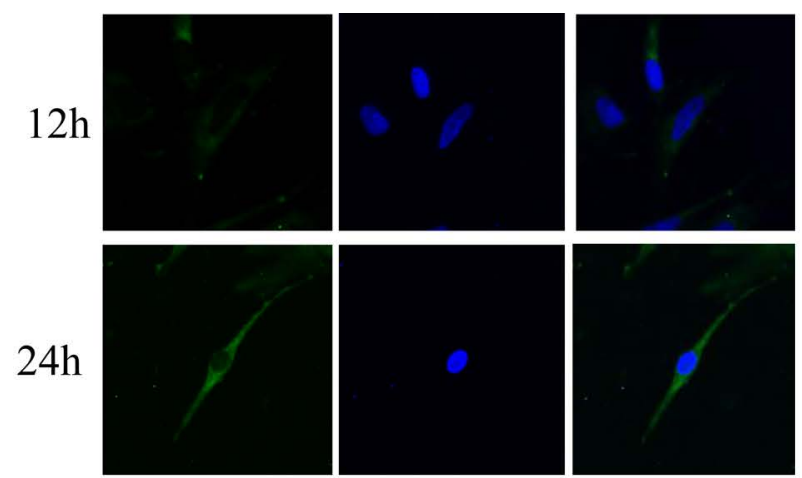

$36 \mathrm{~h}$
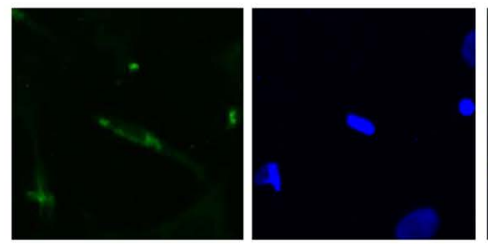

FITC
DAPI

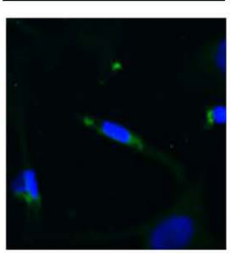

Merge
$48 \mathrm{~h}$
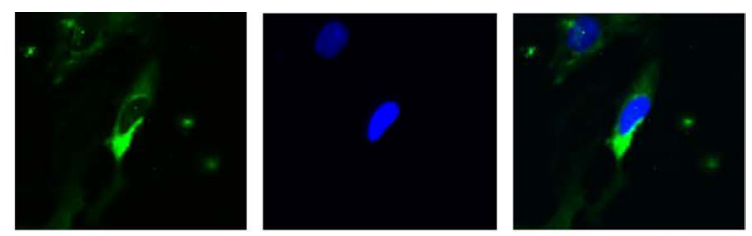

$60 \mathrm{~h}$
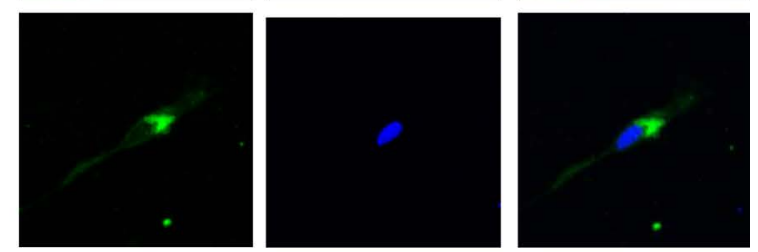

$72 \mathrm{~h}$

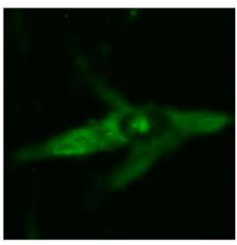

FITC

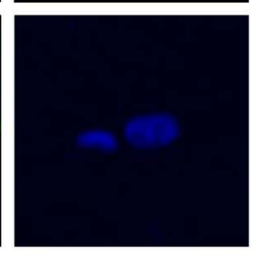

DAPI

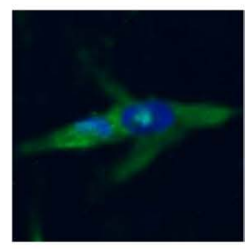

Merge

Fig. 4

Localization of NS2A in DTMUV infected DEF cells

Indirect immunofluorescence was used to analyze the localization of NS2A at different time points during DTMUV infection. FITC (green) was used to show the localization of NS2A, DAPI (blue) was used to indicate the nucleus. Magnification $\times 400$.

At the exponential stage, the cells were induced by IPTG and cultivated at different temperatures and samples were collected at different time points to optimize the expression conditions. As shown in figure lc,d,e, we obtained the highest expression by induction with $0.3 \mathrm{mmol} / 1$ IPTG for $6 \mathrm{~h}$ at $37^{\circ} \mathrm{C}$. After sonication and centrifugation, the supernatants and the insoluble fraction were analyzed by SDS-PAGE and Western blot. The results showed that the recombinant proteins were accumulated in the inclusion bodies in the insoluble fraction (Fig. 2a,b). The purified NS2A protein was verified by SDS-PAGE and Western blot (Fig. 2c, d) (mouse anti-His was used in WB). The mice were immunized with three doses of recombinant protein ( $200 \mu \mathrm{g}$ protein and Freund's adjuvant were used to immunize one mouse). One week after the third immunization, serum was collected. Then, sensitivity was measured by ELISA. In ELISA, we have used purified truncated NS2A protein as antigen. The polyclonal serum was applied in different dilutions and followed by secondary goat anti mouse IgG antibody. The absorbance was measured at 450 $\mathrm{nm}$. The titer of the serum was the highest dilution of the serum where the absorbance ratio of the treatment group and the negative control was greater than or equal to 2 . As shown in Fig. 3a, the titer of the serum was approximately to $1: 128,000$. The specificity of the polyclonal antibodies was tested by Western blot. The recombinant NS2A protein was used for electrophoresis and after transfer it was detected by the polyclonal serum followed by the goat anti mouse IgG as secondary antibody. Polyclonal antibodies specifically recognized the recombinant NS2A protein (Fig. 3b).

As shown in figure 4, the localization of DTMUV NS2A have changed greatly in the infected DEF cells with the increasing time of infection. During the beginning of the infection, we could find only small proportion of fluorescence in the cell. Later, the fluorescence could be seen closer to the nucleus. At the end of infection, the fluorescence was distributed in the whole cell. The changes of the localization were in connection with the life cycle of the virus, because NS2A plays part in the construction of replication complex and assembly of DTMUV. We assume that the localization of NS2A changes with the localization of where replication or assembly of DTMUV takes place. To verify the localization of NS2A within other cell organelles in BHK21 cells, EGFP was linked to NS2A. The plasmids pDsRed2-ER or pDsRed2-Mito were used in this experiment. We found that DTMUV NS2A was localized predominantly within the endoplasmic reticulum (ER) (Fig. 5a). Meanwhile, NS2A did not localize within mitochondria in BHK21 cells (Fig. 5b). Those results are consistent with previous reports.

Altogether, the polyclonal antiserum against 60-150 amino acids long domain of NS2A with high sensitivity and specificity was prepared. By fluorescence imaging, 
(a)
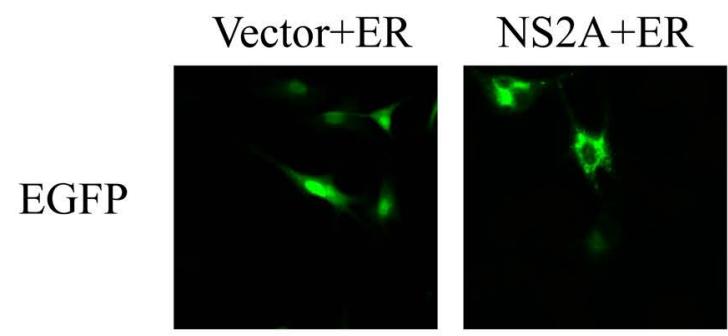

DsRed
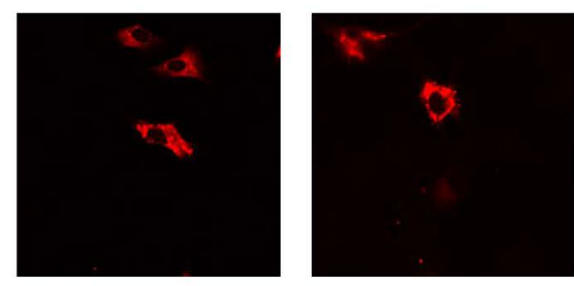

DAPI
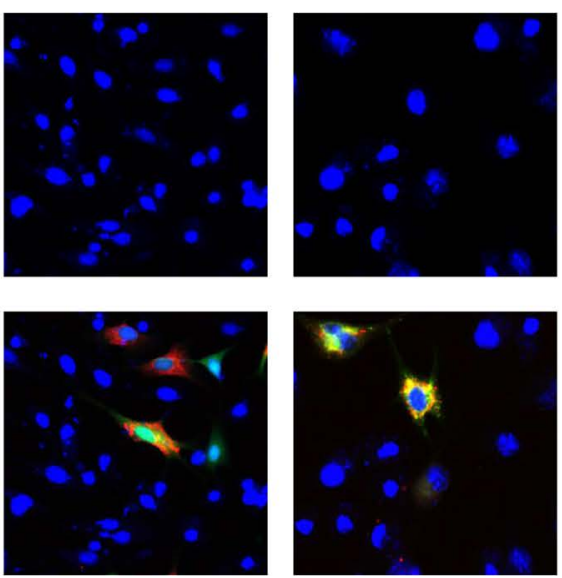

(b)
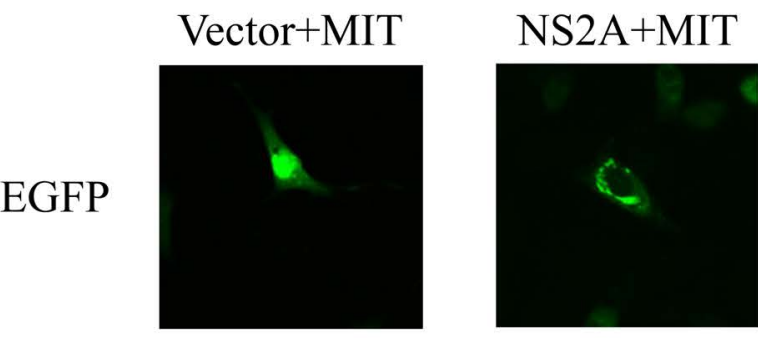

DsRed
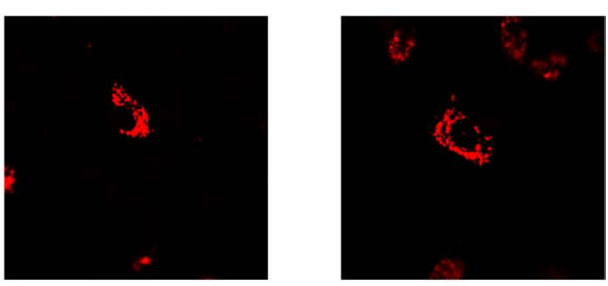

DAPI
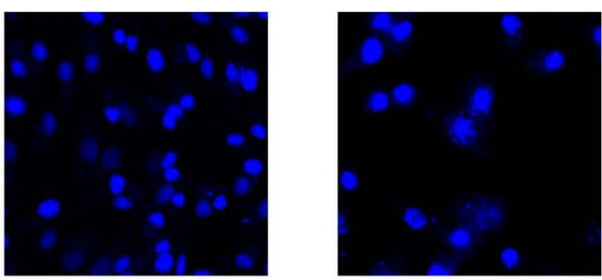

Merge

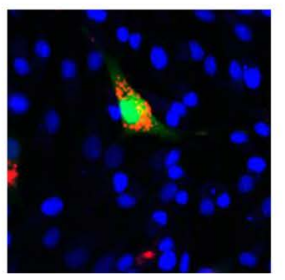

Fig. 5

Localization of DTMUV NS2A within endoplasmatic reticulum or mitochondria in transfected BHK21 cell BHK21 cells were co-transfected with DTMUV EGFP-NS2A plasmid and pDsRed2-ER plasmid (a) or pDsRed2-Mito plasmid (b) $24 \mathrm{~h}$ post transfection. Fluorescence was detected by fluorescence microscope. Magnification $\times 400$.

we have proved that of NS2A was localized within the ER, but not mitochondria in mammalian BHK1 cells. We have shown that DTMUV NS2A might play an important role in the replication of the virus and that our polyclonal antibodies could be a useful tool for further research.

Acknowledgments. We are supported by grants from the National Key R \& D Program (2016YFD0500800), and China Agricultural Research System (CARS-42-17), the SichuanInternational joint research for science and technology (2018HH0098), the Program Sichuan Veterinary Medicine and Drug Innovation Group of China Agricultural Research System (CARS-SVDIP) and the Integration and Demonstration of Key Technologies for Goose Industrial Chain in Sichuan Province (2018NZ0005).

\section{References}

Bhatt S, Gething PW, Brady OJ, Messina JP, Farlow AW, Moyes CL, Drake JM, Brownstein JS, Hoen AG, Sankoh O, Myers MF, George DB, Jaenisch T, Wint GR, Simmons CP, Scott TW, Farrar JJ, Hay SI (2013): The global distribution and burden of dengue. Nature 496, 504-547. https:// doi.org/10.1038/nature12060

Chambers TJ, McCourt DW, Rice CM (1989): Yellow fever virus proteins NS2A, NS2B, and NS4B: identification and partial N-terminal amino acid sequence analysis. Virology 169, 100-109. https://doi.org/10.1016/0042$6822(89) 90045-7$

Kummerer BM, Rice CM (2002): Mutations in the yellow fever virus nonstructural protein NS2A selectively 
block production of infectious particles. J. Virol. 76, 4773-4784. https://doi.org/10.1128/JVI.76.10.47734784.2002

Leung JY, Pijlman GP, Kondratieva N, Hyde J, Mackenzie JM, Khromykh AA (2008): Role of nonstructural protein NS2A in flavivirus assembly. J. Virol. 82, 4731-41. https://doi.org/10.1128/JVI.00002-08

Liu M, Chen S, Chen Y, Liu C, Chen S, Yin X, Li G, Zhang Y (2012): Adapted Tembusu-like virus in chickens and geese in China. J. Clin. Microbiol. 50, 2807-9. https://doi. org/10.1128/JCM.00655-12

Liu WJ, Chen HB, Wang XJ, Huang H, Khromykh AA (2004): Analysis of adaptive mutations in Kunjin virus replicon RNA reveals a novel role for the flavivirus nonstructural protein NS2A in inhibition of beta interferon promoter-driven transcription. J. Virol. 78, 12225-35. https://doi.org/10.1128/JVI.78.22.12225-12235.2004

Liu WJ, Wang XJ, Clark DC, Lobigs M, Hall RA, Khromykh AA (2006): A single amino acid substitution in the West Nile virus nonstructural protein NS2A disables its ability to inhibit alpha/beta interferon induction and attenuates virus virulence in mice. J. Virol. 80, 2396-404. https://doi.org/10.1128/JVI.80.5.2396-2404.2006

Munoz-Jordan JL, Sanchez-Burgos GG, Laurent-Rolle M, GarciaSastre A (2003): Inhibition of interferon signaling by dengue virus. Proc. Natl. Acad. Sci. U S A 100, 14333-8. https://doi.org/10.1073/pnas.2335168100

Shaozhou W, Li C, Zhang Q, Meng R, Gao Y, Liu H, Bai X, Chen Y, Liu M, Liu S, Zhang Y (2015): Duck tembusu virus and its envelope protein induce programmed cell death. Virus Genes 51, 39-44. https://doi.org/10.1007/ s11262-015-1200-2

Su J, Li S, Hu X, Yu X, Wang Y, Liu P, Lu X, Zhang G, Hu X, Liu D, Li X, Su W, Lu H, Mok NS, Wang P, Wang M, Tian K, Gao GF (2011): Duck egg-drop syndrome caused by BYD virus, a new Tembusu-related flavivirus. PLoS One 6, e18106. https://doi.org/10.1371/journal.pone.0018106

Sun X, Wang S, Lin X, Zhao L, Zhang D, Yi C, Sun X, Chen H, Jin M (2017): Proteome analysis of Duck Tembusu virus (DTMUV)-infected BHK-21 cells. Proteomics 17. https:// doi.org/10.1002/pmic.201700033

Tang Y, Diao Y, Yu C, Gao X, Ju X, Xue C, Liu X, Ge P, Qu J, Zhang D (2013): Characterization of a Tembusu virus isolated from naturally infected house sparrows (Passer domesticus) in Northern China. Transbound. Emerg. Dis. 60, 152-8. https://doi.org/10.1111/j.18651682.2012.01328.x

Tu YC, Yu CY, Liang JJ, Lin E, Liao CL, Lin YL (2012): Blocking double-stranded RNA-activated protein kinase PKR by Japanese encephalitis virus nonstructural protein 2A. J. Virol. 86, 10347-58. https://doi.org/10.1128/ JVI.00525-12

Wang J, Lei CQ, Ji Y, Zhou H, Ren Y, Peng Q, Zeng Y, Jia Y, Ge J, Zhong B, Li Y, Wei J, Shu HB, Zhu Q (2016): Duck Tembusu virus nonstructural protein 1 antagonizes IFN-beta signaling pathways by targeting VISA. J. Immunol.197, 4704-4713. https://doi.org/10.4049/jimmunol.1502317

Westaway EG, Mackenzie JM, Kenney MT, Jones MK, Khromykh AA(1997): Ultrastructure of Kunjin virus-infected cells: colocalization of NS1 and NS3 with double-stranded RNA, and of NS2B with NS3, in virus-induced membrane structures. J. Virol. 71, 6650-61. https://doi. org/10.1128/JVI.71.9.6650-6661.1997

Xie X, Gayen S, Kang C, Yuan Z, Shi PY (2013): Membrane topology and function of dengue virus NS2A protein. J. Virol. 87, 4609-22. https://doi.org/10.1128/JVI.02424-12

Zhang W, Chen S, Mahalingam S, Wang M, Cheng A (2017): An updated review of avian-origin Tembusu virus: a newly emerging avian Flavivirus. J. Gen. Virol. 98, 2413-2420. https://doi.org/10.1099/igv.0.000908 